also be successfully employed for further control of the confinement versus loss of parameter space. Clearly, an important challenge, and one that should not to be underestimated, will be devising efficient excitation schemes for these highly confined modes, such as grating-induced phase matching.

Graphene should not only be seen as a mere materials, and hence operating frequency, extension to plasmonics; but also as a fascinating 'substrate' for metalnanoparticle-based plasmonics at visible frequencies. Opportunities for novel biosensing and photodetection schemes are manifold, combining the light-harvesting power of metal-nanostructure optical antennas, with the low-loss electrical characteristics of graphene $e^{8,9}$. This positive outlook for applications also goes hand-in-hand with the potential for fascinating fundamental studies, such as the transfer of hot electrons generated in metallic nanoparticles by plasmon decay directly to the graphene $\mathrm{e}^{10}$, blurring optical and electrical functionalities within the plasmonic nanoantenna framework. Graphene could be just what the plasmonics community has been waiting for - the perfect interface to the two-dimensional world of electromagnetic surface waves.
Stefan A. Maier is at the Blackett Laboratory, Imperial College London, South Kensington, London SW7 2AZ, UK.

e-mail:s.maier@imperial.ac.uk

References

1. Fei, Z. et al. Nature 487, 82-85 (2012).

2. Chen, J. et al. Nature 487, 77-81 (2012)

3. Allen, M. J., Tung, V. C. \& Kaner, R. B. Chem. Re 110, $132-145$ (2010)

4. Giannini, V., Fernández-Domínguez, A. I., Heck, S. C. \& Maier, S. A. Chem. Rev. 111, 3888-3912 (2011).

Schuller J. A. et al Nature Mater 9, 193-204 (2010)

6. Nikitin, A. Y., Guinea, F., Garcia-Vidal, F. J. \& Martin-Moreno, L Phys. Rev. B 84, 161407 (2011).

Vogelgesang, R. \& Dmitriev, A. Analyst 135, 1175-1181 (2010).

8. Echtermeyer, T. J. et al. Nature Commun. 2, 458 (2011).

9. Liu, Y. et al. Nature Commun. 2, 579 (2011)

10. Fang, Z., Liu, Z., Wang, Y., Ajayan, P. M., Nordlander, P. \& Halas, N. J. Nano Lett. 12, 3808-3813 (2012)

\title{
QUASICRYSTALS
}

\section{Tile at random}

The stunning aperiodicity of quasicrystalline systems, once confined to an atomic scale, has recently surfaced on the mesoscale in a number of softmatter materials. It turns out that certain liquid-crystal, polymer and colloidal systems are capable of supporting quasicrystals - brightening the prospects for understanding this intriguing order, and providing new avenues for research into viable photonic bandgap materials. But the fine control afforded by these systems is hampered by their structural complexity. Now, Changhong Xiao and co-workers have succeeded in synthesizing a class of mesoporous silica materials exhibiting dodecagonal symmetry - achieving the first hard-matter quasicrystal in the mesoscale range (Nature 487, 349-353; 2012).

The dendritic liquid-crystal system that exhibits dodecagonal quasicrystals does so under the same conditions required to synthesize two of the most commonly observed structures in mesoporous silica. Motivated by this parallel, Xiao et al. set about investigating the idea that quasicrystals might also appear in the silica system. The two crystal structures in question comprise different kinds of Voronoi polyhedron, and are generated by tuning alkalinity during synthesis. A third structure can be induced at intermediate alkalinity.

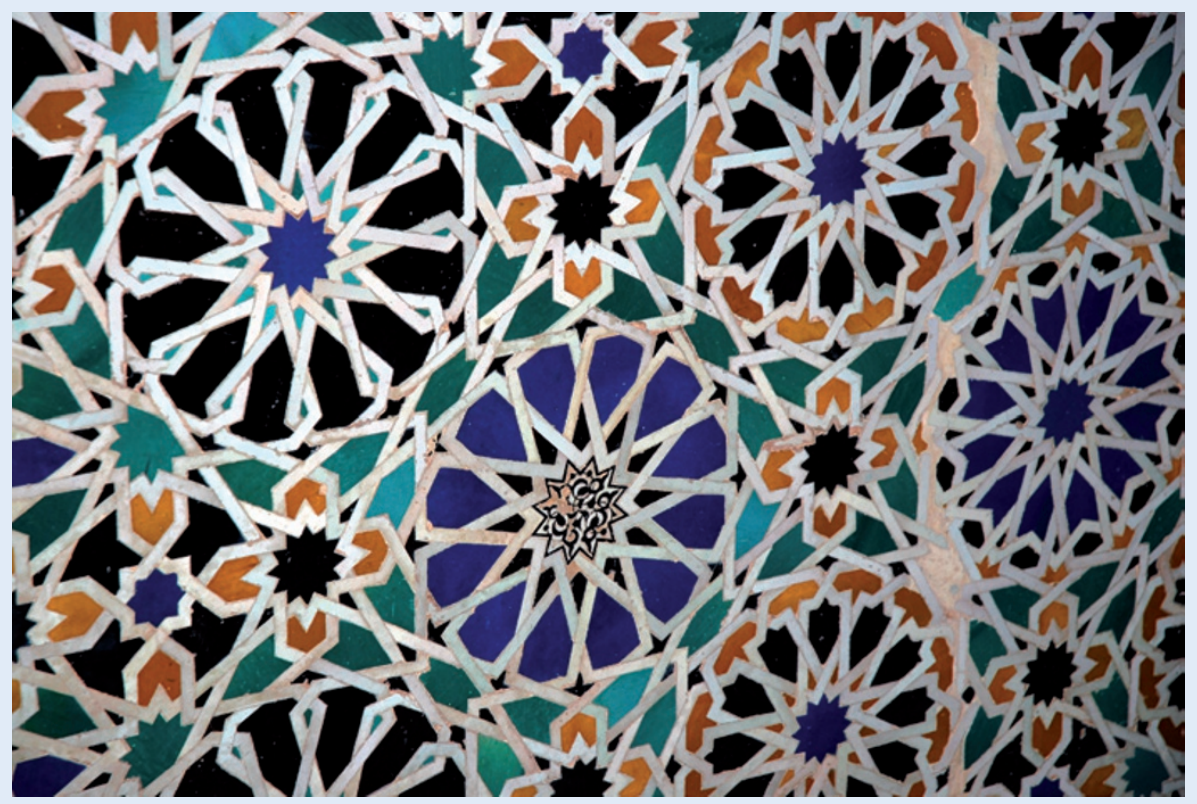

Using transmission electron microscopy, Xiao et al. found that the synthesis process also gave rise to a dodecagonal quasicrystalline arrangement.

Projections of the three structures resemble periodic arrays of squares and equilateral triangles. The group's investigation revealed that the structure associated with intermediate alkalinity coexists with domains of the other two structures - producing aperiodic patterns much like the exquisite tiling patterns (pictured) that have become emblematic of the study of quasicrystals. Electron diffraction patterns also showed signatures of 12 -fold symmetry that, together with prism-like morphology, betray the presence of dodecagonal order within their samples.

Xiao et al. interpreted their findings using a non-equilibrium formation process, consistent with the observations from the microscopy study. The process begins with a clustered tiling of squares and triangles, which grows via a random selection of neighbouring vertices. The authors' model builds on a stochastic framework originally developed to study the growth of tumours and the spread of bacteria. It suggests that an exclusively triangular tiling can be avoided only in the case that adjacent triangles repel one another. Tuning the repulsive interactions in the model induces transformations between the crystalline structures, and beyond to a quasicrystalline tiling - a result in line with the fact that these transformations are regulated by changes in alkalinity. 